\title{
Five roadblocks to finding appropriate OCD treatment
}

\section{Opinion}

With OCD we often see that finding appropriate treatment can be a stressful process. The primary goal with OCD treatment is to first find a professional who has the skill to make an accurate diagnosis and then to find a trained professional to provide appropriate treatment. It is important for you to know that OCD is treated most effectively with Cognitive Behavioral Therapy (or CBT) using primarily Exposure Response prevention or E/RP.

When seeking treatment for OCD it is beneficial to know common deterrents to treatment. Here are 5 common problems that interfere or block connecting with an effective treatment for OCD.

1. The sufferer may not be aware that they have OCD even though they are clear that something is wrong.

2. Sufferers may be in treatment but have been misdiagnosed and their OCD is being viewed as something other than OCD.

3. The sufferer may be stuck in a ritual cycle and not know what to do or may be too afraid to stop the ritual for fear of something bad happening.

4. Sufferers can feel safe with their rituals and they know that treatment will challenge that.

5. Sufferers may find it too difficult or embarrassing to admit the content of their specific obsessions. The content of OCD can present as out control, uncomfortably odd, grossly illogical or perverse. Individuals would often rather suffer the anxiety of the OCD than the humiliation of admitting the content. Worse yet, they may fear that an admission would cause a rejection and loss of all that they love in this life. For instance, they may be obsessing by asking themselves "What if I'm gay", or "What if I become violent and harm or kill someone or "What if I'm a pedophile"?"

While to the general public OCD is most often seen as a problem related to hand washing, checking, and cleaning. The list of possible contents for OCD is essentially endless, produced by an able imagination. If you are creative and can imagine a fear, you can most certainly imagine your worst fear and obsess about it.

The 5 reasons I've named discourage many individuals from seeking help. As a result, it can take years to make it to an effective treatment. This is not an exaggeration. One estimate reports that it takes 14-17 years from onset of symptoms to finding appropriate treatment.

In addition, finding appropriate treatment has its difficulties as well. Here are a couple of issues you may encounter in finding a specialist.

The number of OCD trained professionals is growing, but at present there are still too few therapists with specific training or adequate experience treating OCD.

Most psychotherapists' practices as generalists treating a wide range of mental health problems but are not trained to recognize or treat $\mathrm{OCD}$
Volume 9 Issue 5 - 2018

\section{Harold Kirby \\ Mindful Stress \& Anxiety Management Center of Philadelphia, USA}

Correspondence: Harold Kirby, Mindful Stress \& Anxiety Management Center of Philadelphia, USA, Email hpkirby@comcast.net

Received: August 16, 2015 | Published: October 10, 2018

When looking for a provider, many sufferers who are inexperienced with treatment believe that therapists are one size fits all and choose an individual who has limited knowledge of E/RP, in particular.

OCD treatment is somewhat different. While many psychological disorders respond to a generalist approach. Working with OCD, we find that the therapist must understand the specifics of the disorder and the treatment strategies that work. A standard generalist psychological approach, especially if it is mostly supportive in nature, will not work, and it can complicate the problem of OCD by unwittingly providing reassurance.

The reassurance unfortunately becomes fuel for the OCD rituals, keeping the sufferer stuck.

For example, a supportive approach often utilizes reassurance as a means of showing compassion. A well-meaning therapist may reassure a young man who is obsessing about the possibility of being gay that it is not likely because he has always preferred women.

This effort is aimed at helping the individual feel better in the moment and is based in evidence but in the final analysis it will stall his ability to get better by becoming a ritual.

Thank you, he will say until...the next obsession...He will ask... how do they really know? What if I am gay or even bisexual, which begins a new cycle of obsession?

Compassion for persons suffering with OCD is found in helping them face their worst fears produced by the OCD and learning to cope with those fears. It is not helped by reassuring them that nothing bad is likely to happen or that what they fear will never be true.

While OCD treatment is specialized, it is not complex or beyond the grasp of the general clinical practitioner or sufferer. In fact, learning to do exposure response prevention is not difficult at all. The difficult part is being experienced enough to target the problem accurately and to motivate people to do the anxiety-producing treatment that is required.

In other words, the specialist must understand and have experienced the counterintuitive nature of OCD and be skilled at helping the sufferer live with significant anxiety. Learning as they cope with their fear to move to accepting that no one can completely eliminate risks in living but we can cope with them and have a life we value. 
It is not common knowledge that OCD requires a CBT treatment using primarily exposure response prevention. To have good treatment outcomes, it is imperative to find a person who specializes in the treatment of OCD and has worked with many cases over a period of time.

Often a sufferer is not aware of the unique nature of his/her disorder, and in desperation, will commit to any form of treatment intervention for hope of improvement and relief. To an inexperienced person, therapy is therapy, right? As often happens, the individual becomes attached to the therapist, their kindness and understanding. This attachment, without appropriate treatment strategies, however, leads to working in an ineffective way that will confound even the best efforts.

Sufferers who are not being treated using primarily exposure response prevention often spend time doing treatment that isn't helpful or worse, ultimately end up believing that are untreatable because of the repeated poor outcomes. Unfortunately, then, some sufferers simply give up because they have not found or received effective treatment.
Please don't give up. If you are seeking effective treatment, I hope that you will start or restart your efforts in getting better. Both the AMA and APA recommend exposure response prevention as a firstline psychological treatment for OCD. There are people who devote their entire practice to treating OCD and related disorders. There is effective help.

If you or someone you care about has OCD, please start your search at IOCDF.org or ADAA.org to locate an individual in your area who is qualified and experienced in working with OCD. Good luck and stay strong searching until you find the right therapist. It will be worth it!

\section{Acknowledgements}

None.

\section{Conflict of interest}

The author declares that there is no conflict of interest. 